\title{
An integrated diversity and fusion framework for binary consensus over fading channels
}

\author{
Mehrzad Malmirchegini ${ }^{*}$ and Yasamin Mostofi
}

\begin{abstract}
In this paper, we consider a cooperative network that is trying to reach consensus on the occurrence of an event by communicating over not fully connected and time-invariant network topologies with fading channels. We first discuss the fusion and diversity decision-making strategies over time-invariant network topologies and shed light on the underlying trade-offs. We then propose an integrated diversity and fusion framework. Our approach properly takes advantage of both fusion to enable information flow and diversity to increase robustness to link errors. We mathematically analyze the proposed framework and show how the network achieves accurate consensus asymptotically. To show an example, we then utilize the proposed framework over regular ring lattice networks. Our theoretical and simulation results indicate that the proposed technique improves the consensus performance considerably.
\end{abstract}

\section{Introduction}

Cooperative decision-making over sensor networks has gotten considerable interest in recent years. These networks have a variety of applications such as environmental monitoring, target tracking, and surveillance. Consider the scenario where a network of nodes, distributed in a harsh environment, aims to cooperatively accomplish a task. Each node has limited local capabilities and can therefore only accomplish the task in a cooperative manner. In this paper, we are interested in group agreement problem, where a group of agents need to reach consensus on the value of a parameter of interest. The consensus problems can be categorized into two main groups: estimation consensus and detection consensus. Estimation consensus refers to problems in which each agent has an estimate of the parameter of interest, where the parameter of interest can take values over an infinite set or an unknown finite set. For instance, it may be of interest that all the mobile agents that started in different directions reach an agreement on their asymptotic headings in a cooperative multi-agent network [1]. Recently, there has been considerable interest in estimation consensus problems from the signal processing and communication

*Correspondence: mehrzad@ece.ucsb.edu

Electrical and Computer Engineering Department, University of California, Santa Barbara, CA, USA community, with more emphasis on link uncertainties [2-4].

Detection consensus, on the other hand, refers to problems in which the parameter of interest takes values from a finite known set. Then the update protocol, which each agent will utilize, becomes nonlinear. By binary consensus [5], we refer to a subset of detection consensus problems where the network is trying to reach consensus over a binary parameter. For instance, in a cooperative fire detection scenario, each node has an initial opinion as to if there is a fire or not. However, as a network, they may act based only on the majority vote. Therefore, the goal of the network is for each node to reach consensus over the majority of initial votes. Another application of binary consensus is in cooperative spectrum sensing in cognitive radio networks. In this scenario, the secondary users communicate with each other in order to reach consensus on busy or idle status of the primary user, which is a binary value [6].

\subsection{Related work and our contribution}

While there exists a rich literature on estimation consensus, detection consensus problems only recently started to receive attention. In [7], the authors consider convergence in a detection consensus setup over perfect channels with repeated sensing and known probabilistic sensing models. In [8], the authors consider a distributed hypothesis testing problem over perfect communication channels to

\section{Springer}

(c) 2013 Malmirchegini and Mostofi; licensee Springer. This is an Open Access article distributed under the terms of the Creative Commons Attribution License (http://creativecommons.org/licenses/by/2.0), which permits unrestricted use, distribution, and reproduction in any medium, provided the original work is properly cited. 
which they refer to as belief consensus. They consider the case where each node transmits its belief (conditional probability) to other nodes. As a result, their problem immediately takes the form of the traditional average estimation consensus for which a rich literature exists.

In [5,9], the binary consensus scenario is considered. In this scenario, each node in the network has an opinion regarding the occurrence of an event. The nodes will then communicate over the network topology. The goal for every node is to reach the majority of the initial votes without any prior knowledge on the sensing qualities. Authors in [9] characterized phase transition of such a binary consensus problem in the presence of a uniformly distributed communication noise. In most applications, however, the agents will communicate their values wirelessly and will experience Gaussian receiver noise as opposed to a uniformly distributed noise. In [5], we considered reaching binary consensus over regular network topologies (all nodes have the same number of neighbors) with additive white Gaussian noise channels. We characterized the transient behavior of the network probabilistically. We showed that in the presence of noise, the network state is asymptotically memoryless, i.e., independent of the initial state. This is undesirable since the group agreement is not related to the initial state of the system and is merely a function of channel errors.

In [10], we studied the binary consensus over a fully connected network topology with fading channels. We proposed a novel consensus-seeking protocol that utilizes information of link qualities. We showed that by incorporating the information of link qualities, the network will be in consensus with a higher probability but still holds the undesirable asymptotic behavior. In [11], we considered binary consensus over rapidly changing network topologies with fading channels. We mathematically characterized the impact of fading, noise, network connectivity and time-varying topology on consensus performance.

In this paper, we consider the binary consensus problem over the general time-invariant network topologies (not necessarily fully connected) with fading channels. We study two decision-making strategies that differ in terms of using the available transmissions: fusion and diversity. In the first strategy, the given resources are used to increase the flow of information in the network, whereas the second strategy aims to increase robustness to link error by channel coding [11]. We characterize the underlying trade-offs between these two strategies for binary consensus over a not fully connected and time-invariant network topology. In particular, we show that fusionbased scheme results in an asymptotic memoryless behavior, which is not desirable. Furthermore, diversity-based scheme only outperforms fusion-based scheme, when the main bottleneck is link quality and the network has a good connectivity. The main contribution of this paper is to propose a framework that keeps the benefits of both fusion and diversity strategies, in terms of the network information flow and link error robustness, for binary consensus over time-invariant network topologies with fading channels. We mathematically analyze the proposed framework and show that it achieves accurate consensus asymptotically. The proposed framework solves the undesirable memoryless behavior of the network consensus and results in a drastic performance improvement.

\section{Binary consensus problem over time-invariant topologies with ideal communication links}

In this section, we introduce the binary consensus problem over not fully connected and time-invariant network topologies. Furthermore, we assume ideal communication links. Studying this case allows us to focus solely on the impact of information flow in the network and characterize a benchmark for the performance of binary consensus algorithms over non-ideal communication links.

Consider a cooperative network of $M$ nodes $^{\mathrm{a}}$ that are trying to reach consensus over the occurrence of an event. Each node has its own initial decision, based on its onetime sensing. The goal of the network is for each node to reach a decision that is equal to the majority of the initial votes. For instance, in a cooperative fire detection scenario, each node has an initial opinion as to if there is a fire or not. However, as a network, they may act based only on the majority vote. Therefore, it is desirable that every node reaches the majority of the initial votes without a group leader. As it may happen in realistic scenarios, the nodes may not have any information on their own or the others' sensing quality. Therefore, the main goal is that each node reaches the majority of the initial votes. Considering sensing quality of the nodes is among possible extensions of this work.

In order to achieve this, each node will transmit its current decision to other nodes. ${ }^{\mathrm{b}}$ We model the underlying network as an undirected graph $G(\mathcal{V}, \mathcal{E})$, where $\mathcal{V}=\{1, \ldots, M\}$ represents the vertex set and $\mathcal{E}$ is the link set (the set of available communication links among the nodes). Each node will then revise its current vote based on the received information. This process will go on for a while. We say that accurate consensus is achieved if each agent reaches the majority of the initial votes [5]. For instance, if $70 \%$ of the nodes start voting one and they all end up voting one, we have accurate consensus. Let $\tilde{b}_{i}^{0} \in\{0,1\}$ represent the initial vote of the $i$ th node, where $\tilde{b}_{i}^{0}=1$ indicates that the $i$ th agent initially decides that the event occurred, whereas $\tilde{b}_{i}^{0}=0$ denotes otherwise. Consider the binary consensus problem over ideal communication links. In this scenario, each node receives the votes of its neighbors over the ideal communication links. It then fuses all the received information 
and updates its vote based on the majority of its neighbors' votes.

Let $\tilde{b}_{i}^{u}$ denote the $i$ th node's vote after $u$ fusion steps over ideal communication links. We have,

$$
\tilde{b}_{i}^{u}=\Upsilon_{\text {ideal }}\left(\frac{1}{1+\left|\mathcal{N}_{i}\right|}\left[\tilde{b}_{i}^{u-1}+\sum_{j \in \mathcal{N}_{i}} \tilde{b}_{j}^{u-1}\right], \tilde{b}_{i}^{u-1}\right)
$$

where $\mathcal{N}_{i}$ denotes the neighbor set of the $i$ th node, and the decision-making function for the binary consensus over ideal communication links, $\Upsilon_{\text {ideal }}(.$, .), is defined as follows:

$$
\Upsilon_{\text {ideal }}(x, \xi) \triangleq \begin{cases}1 & x>0.5 \\ \xi & x=0.5 \\ 0 & x<0.5\end{cases}
$$

Figure 1 shows a network of $M=17$ nodes trying to reach consensus over a 6-regular ring lattice. ${ }^{\mathrm{C}}$ In this topology, each node receives information from its neighbors over ideal communication links. As can be seen, all the nodes reach the majority of initial votes at the second level of fusion. Let $D^{u}=\left\{\tilde{b}_{1}^{u}, \tilde{b}_{2}^{u}, \ldots, \tilde{b}_{M}^{u}\right\}$ denote the network state after $u$ fusion steps. Define

$$
u_{G}\left(D^{0}\right) \triangleq \min \left\{u \mid D^{u} \text { is an accurate consensus state }\right\}
$$

For instance, for the example of Figure 1 , we have $D^{0}=$ $\{1,0,0,1,0,1,0,1,1,1,0,0,0,1,1,0,1\}$ and $u_{G}\left(D^{0}\right)=2$. We then define $\mathcal{D}_{G} \triangleq\left\{D^{0} \mid u_{G}\left(D^{0}\right)<\infty\right\}$ and $u_{G}=$ $\max _{D^{0} \in \mathcal{D}_{G}} u_{G}\left(D^{0}\right), u_{G}$ is only a function of graph connectivity and represents the maximum number of required fusion levels in order to achieve accurate consensus if $D^{0} \in \mathcal{D}_{G}$. For instance, for the fully connected graph, i.e., each node is connected to all the nodes in the network, we have $u_{G}=1$. In the next section, we consider the binary consensus problem over non-ideal communication links. We discuss different strategies in terms of information flow and robustness to link error and characterize the underlying trade-offs.

\section{The underlying trade-offs between information flow and link error robustness}

In a realistic scenario, each node transmits its decision to other nodes over non-ideal communication links. The transmissions occur over fading channels and are furthermore corrupted by the receiver noise. Let $r_{j, i}(k)$ represent the fading coefficient of the link from node $j$ to node $i$ at time $k$. Similarly, $n_{j, i}(k)$ denotes the receiver noise at the $k$ th time step in the transmission from the $j$ th node to the $i$ th one. $n_{j, i}(k)$ is zero-mean Gaussian with the variance of $\sigma_{j, i}^{2}$. We take the receiver noise of the receptions of different nodes to be uncorrelated. Let $b_{j, i}(k)$ represent the reception of the $i$ th node from the transmission of the $j$ th one at the $k$ th time step. We have the following if there exists a link from the $j$ th node to the $i$ th one: $^{\mathrm{d}}$ $b_{j, i}(k)=r_{j, i}(k) b_{j}(k)+n_{j, i}(k)$, where $b_{j}(k)$ denotes the $j$ th node's vote at time $k$. We assume that each receiver can learn the fading coefficient of each of its receptions and utilize it in the decision-making process. Furthermore, we consider the case where the fading coefficients are uncorrelated among different nodes and in different time steps. Each agent will then update its assessment based on the received information. We next discuss fusion and diversity decision-making strategies for binary consensus problem over not fully connected and time-invariant network topologies and characterize the underlying trade-offs.

\subsection{Fusion case}

In this case, each node fuses its received information in every time step to form its new opinion, which it will then

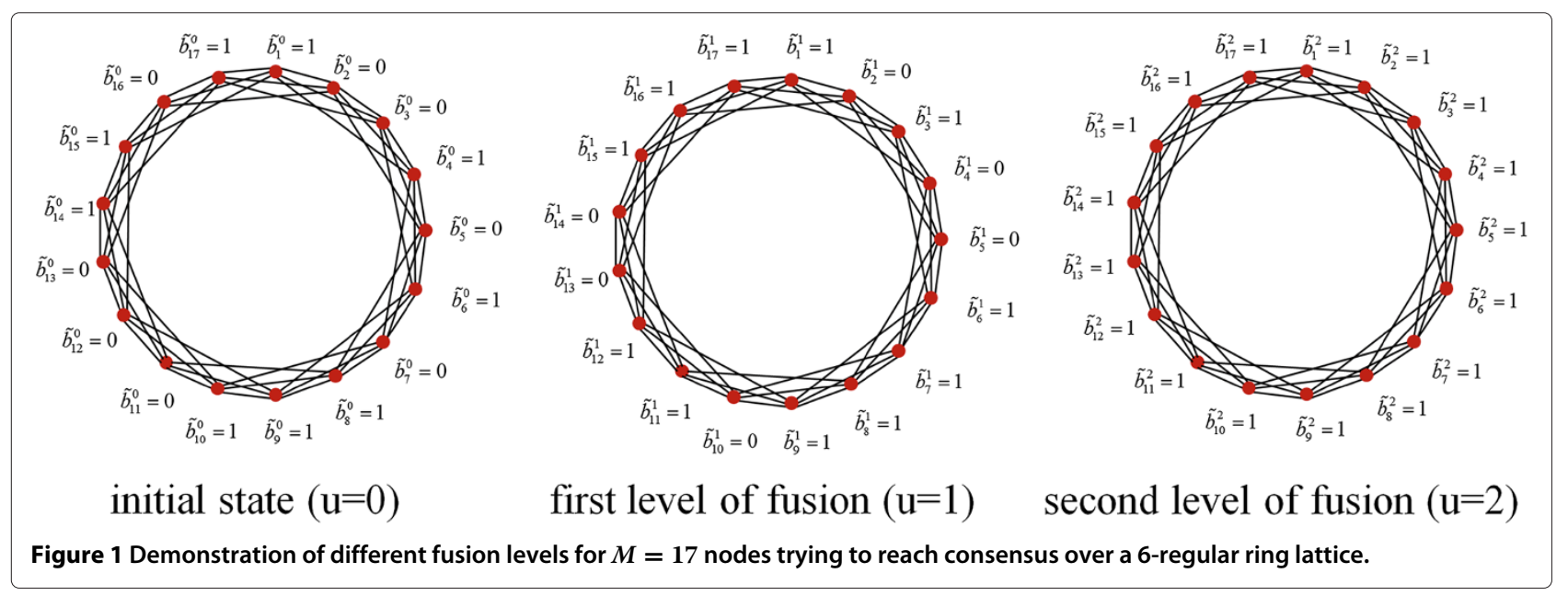


send to other nodes. In this manner, each node helps to propagate the information of other nodes in the network. This strategy is suitable, in particular when the graph connectivity is low, as it creates virtual links between nodes. In [10], we proposed a fusion-based protocol that utilizes information of link qualities and noise variance. In this approach, each node tries to estimate the majority of its neighbors' votes based on the best affine estimation (BAE) of the sum of its neighbors' votes. Let $\psi_{i}(k)=\sum_{j \in \mathcal{N}_{i}} b_{j}(k)$ represent the sum of the votes of the $i$ th node's neighbors. Then the $i$ th node estimates $\psi_{i}(k)$ using the best affine unbiased function of the received information: $\hat{\psi}_{i}(k)=$ $\sum_{j \in \mathcal{N}_{i}} \alpha_{j, i}(k) b_{j, i}(k)+\beta_{j, i}(k)$ where

$$
\begin{aligned}
& \alpha_{j, i}(k)=\frac{r_{j, i}(k)}{r_{j, i}^{2}(k)+\frac{\sigma_{j, i}^{2}}{q_{j}(k)\left(1-q_{j}(k)\right)}} \text { and } \\
& \beta_{j, i}(k)=\left(1-\alpha_{j, i}(k) r_{j, i}(k)\right) q_{j}(k),
\end{aligned}
$$

and $q_{j}(k)=\mathbf{E}\left[b_{j}(k)\right]$ (readers are referred to [10] for more information). The $i$ th node will then update its decision as follows:

$$
\begin{aligned}
b_{i}(k+1)= & \Upsilon_{\text {fading, }\left|\mathcal{N}_{i}\right|+1}\left(\frac{1}{1+\left|\mathcal{N}_{i}\right|}\right. \\
& \left.\times\left[b_{i}(k)+\sum_{j \in \mathcal{N}_{i}}\left(\alpha_{j, i}(k) b_{j, i}(k)+\beta_{j, i}(k)\right)\right], b_{i}(k)\right),
\end{aligned}
$$

where $b_{i}(0)=\tilde{b}_{i}^{0}$ for $1 \leq i \leq M$. The decision-making function for the binary consensus over fading communication links, $\Upsilon_{\text {fading, }, M}(.,$.$) , is defined as follows:$

$$
\begin{aligned}
& \Upsilon_{\text {fading, } M=2 j}(x, \xi) \triangleq\left\{\begin{array}{ll}
1 & x>\frac{1}{2}+\frac{1}{2 M} \\
\xi & \left|x-\frac{1}{2}\right| \leqslant \frac{1}{2 M} \\
0 & x<\frac{1}{2}-\frac{1}{2 M}
\end{array}\right. \text { and } \\
& \Upsilon_{\text {fading, } M=2 j+1}(x, \xi) \triangleq \begin{cases}1 & x \geqslant \frac{1}{2} \\
0 & x<\frac{1}{2}\end{cases}
\end{aligned}
$$

It can be seen that Equation 3 assumes that the knowledge of $q_{j}(k)$ is available at the receiver. If the $i$ th node does not have an estimate of $q_{j}(k)$, it will assume that $q_{j}(k)=0.5$. We refer to this case as basic BAE.

In section III-B of [11], we mathematically analyzed the asymptotic behavior of the basic BAE for binary consensus over fully connected network topology. We showed that the network loses its memory of the initial state asymptotically due to the impact of link errors, which is undesirable. The same analysis can also be extended to the case of not fully connected network topologies. Therefore, the fusion approach for binary consensus over not fully connected and time-invariant network topology and in the presence of noise and fading suffers from asymptotic memoryless behavior. Next, we discuss the diversity-based scheme, which is more robust to the link error.

\subsection{Diversity case}

In this part, we consider another strategy in which each node uses its transmissions to repeat its initial vote. Consider the case where the network is given $K+1$ transmissions to reach an agreement. Each node can use all of its transmissions to repeat its initial vote and only fuses the received information afterward. This strategy can, in particular, be useful in reducing the impact of link errors. Let $Z_{j, i}(K)=\left[b_{j, i}(0), \ldots, b_{j, i}(K)\right]^{T}$ denote the receptions of $i$ th node from the $j$ th node in $K+1$ transmissions. Define $R_{j, i}(K)=\left[r_{j, i}(0), \ldots, r_{j, i}(K)\right]^{T}$ and $N_{j, i}(K)=$ $\left[n_{j, i}(0), \ldots, n_{j, i}(K)\right]^{T}$. For the diversity case, all nodes use their transmissions to repeat their initial votes, i.e., $b_{j}(k)=$ $\tilde{b}_{j}^{0}$ for $0 \leq k \leq K$. Then we have the following, considering all the receptions of the $i$ th node from the $j$ th node: $Z_{j, i}(K)=\tilde{b}_{j}^{0} R_{j, i}(K)+N_{j, i}(K)$. The $i$ th node can then estimate $\tilde{b}_{j}^{0}$ by using the best affine unbiased function of the received information: $\hat{b}_{j}^{0}(K)=\rho_{j, i}^{T}(K) Z_{j, i}(K)+\eta_{j, i}(K)$. To ensure an unbiased estimator, we should have, $\mathbf{E}\left[\hat{b}_{j}^{0}(K)\right]=$ $\mathbf{E}\left[\tilde{b}_{j}^{0}\right] \Rightarrow \eta_{j, i}(K)=\tilde{q}_{j}^{0}\left(1-\rho_{j, i}^{T}(K) R_{j, i}(K)\right)$, where $\tilde{q}_{j}^{0}=$ $\mathbf{E}\left[\tilde{b}_{i}^{0}\right]$. Let $\zeta_{j, i}(K)=\mathbf{E}\left[\left(\hat{b}_{j}^{0}(K)-\tilde{b}_{j}^{0}\right)^{2}\right]$ denote the corresponding estimation error variance. We then have the following optimization problem:

$$
\begin{aligned}
\rho_{j, i}(K) & =\arg \min \zeta_{j, i}(K), \\
\text { subject to } \eta_{j, i}(K) & =\tilde{q}_{j}^{0}\left(1-\rho_{j, i}^{T}(K) R_{j, i}(K)\right) .
\end{aligned}
$$

We have

$$
\begin{aligned}
\zeta_{j, i}(K)= & E\left[\left(\hat{b}_{j}^{0}(K)-\tilde{b}_{j}^{0}\right)^{2}\right] \\
= & E\left[\left(\left(\tilde{b}_{j}^{0}-\tilde{q}_{j}^{0}\right) \rho_{j, i}^{T}(K) R_{j, i}(K)-\left(b_{j}(0)-\tilde{q}_{j}^{0}\right)\right.\right. \\
& \left.\left.+\rho_{j, i}^{T} N_{j, i}(K)\right)^{2}\right] \\
= & \rho_{j, i}^{T}(K)\left(\tilde{q}_{j}^{0}\left(1-\tilde{q}_{j}^{0}\right) R_{j, i}(K) R_{j, i}^{T}(K)+\sigma_{j, i}^{2} I_{K \times K}\right) \rho_{j, i}(K) \\
& -2 \tilde{q}_{j}^{0}\left(1-\tilde{q}_{j}^{0}\right) \rho_{j, i}^{T}(K) R_{j, i}(K)+\tilde{q}_{j}^{0}\left(1-\tilde{q}_{j}^{0}\right) .
\end{aligned}
$$


By noting that Equation 7 is a convex function of $\rho_{j, i}(K)$, we have

$$
\begin{aligned}
& \rho_{j, i}(K)=\frac{\tilde{q}_{j}^{0}\left(1-\tilde{q}_{j}^{0}\right)}{\sigma_{j, i}^{2}} \\
& \times\left(\frac{\tilde{q}_{j}^{0}\left(1-\tilde{q}_{j}^{0}\right)}{\sigma_{j, i}^{2}} R_{j, i}(K) R_{j, i}^{T}(K)+I_{K \times K}\right)^{-1} R_{j, i}(K) \\
& =\frac{\tilde{q}_{j}^{0}\left(1-\tilde{q}_{j}^{0}\right)}{\sigma_{j, i}^{2}}\left(I_{K \times K}-\frac{\frac{\tilde{q}_{j}^{0}\left(1-\tilde{q}_{j}^{0}\right)}{\sigma_{j, i}^{2}}}{1+\frac{\tilde{q}_{j}^{0}\left(1-\tilde{q}_{j}^{0}\right)}{\sigma_{j, i}^{2}}\left\|R_{j, i}(K)\right\|^{2}}\right. \\
& \left.\times R_{j, i}(K) R_{j, i}^{T}(K)\right) R_{j, i}(K) \\
& =\frac{1}{\frac{\sigma_{j, i}^{2}}{\tilde{q}_{j}^{0}\left(1-\tilde{q}_{j}^{0}\right)}+\left\|R_{j, i}(K)\right\|^{2}} R_{j, i}(K),
\end{aligned}
$$

and $\eta_{j, i}(K)=\tilde{q}_{j}^{0}\left(1-\rho_{j, i}^{T}(K) R_{j, i}(K)\right)$. We then have the following for the estimation error variance after $K+1$ transmissions: $\zeta_{j, i}(K)=\frac{\sigma_{j, i}^{2}}{\frac{\sigma_{j, i}^{2}}{\tilde{\tilde{q}}_{j}^{0}\left(1-\tilde{q}_{j}^{0}\right)}+\left\|R_{j, i}(K)\right\|^{2}}=$ $\frac{1}{\frac{1}{\tilde{q}_{j}^{0}\left(1-\tilde{q}_{j}^{0}\right)}+\sum_{k=0}^{K} \mathrm{CNR}_{j, i}(k)}$, where $\mathrm{CNR}_{j, i}(k)=\frac{r_{j, i}^{2}(k)}{\sigma_{j, i}^{2}}$ denotes the channel-to-noise ratio for the link from node $j$ to node $i$ at time $k$. We then have the following decision-making function:

$$
\begin{aligned}
b_{i}(K+1)= & \Upsilon_{\text {fading, }\left|\mathcal{N}_{i}\right|+1} \\
& \times\left(\frac{1}{1+\left|\mathcal{N}_{i}\right|}\left(b_{i}(0)+\sum_{j \in \mathcal{N}_{i}} \hat{b}_{j}^{0}(K)\right), b_{i}(0)\right) \\
= & \Upsilon_{\text {fading, }\left|\mathcal{N}_{i}\right|+1}\left(\frac { 1 } { 1 + | \mathcal { N } _ { i } | } \left(b_{i}(0)\right.\right. \\
& \left.\left.+\sum_{j \in \mathcal{N}_{i}} \rho_{j, i}^{T}(K) Z_{j, i}(K)+\eta_{j, i}(K)\right), b_{i}(0)\right),
\end{aligned}
$$

where $b_{i}(k)=\tilde{b}_{i}^{0}$ for $0 \leq k \leq K$.

Lemma 1. Consider binary consensus over a not fully connected network topology. Under the assumption of i.i.d. Rayleigh fading channels, $C N R_{j, i}(k)$ are independent and identically distributed (i.i.d.) exponential random variables with $\overline{C N R}=\mathbf{E}\left\{C N R_{j, i}(k)\right\}$. Then the dynamical system, based on the decision-making function of 8 , will asymptotically reach $D^{1}=\left\{\tilde{b}_{1}^{1}, \tilde{b}_{2}^{1}, \ldots, \tilde{b}_{M}^{1}\right\}$, where $D^{1}$ represents the network state after one level of fusion over ideal communication links.
Proof. It can be easily confirmed that if $\left|b_{i}(0)+\sum_{j \in \mathcal{N}_{i}} \hat{b}_{j}^{0}(K)-\sum_{j \in \mathcal{N}_{i} \bigcup\{i\}} \tilde{b}_{j}^{0}\right| \leq \frac{1}{2}$, then we have $\Upsilon_{\text {fading, } M}\left(\frac{1}{M}\left[b_{i}(0)+\sum_{j \in \mathcal{N}_{i}} \hat{b}_{j}^{0}(K)\right], b_{i}(0)\right)=$ $\Upsilon_{\text {ideal }}\left(\frac{1}{M} \sum_{j \in \mathcal{N}_{i} \bigcup\{i\}} \tilde{b}_{j}^{0}, \tilde{b}_{i}^{0}\right)$, which results in $b_{i}(K+1)=$ $\tilde{b}_{i}^{1}$. Therefore, we have,

$$
\begin{aligned}
\operatorname{prob} & \left(b_{i}(K+1) \neq \tilde{b}_{i}^{1}\right) \\
& \leq \operatorname{prob}\left(\left|b_{i}(0)+\sum_{j \in \mathcal{N}_{i}} \hat{b}_{j}^{0}(K)-\sum_{j \in \mathcal{N}_{i} \bigcup\{i\}} \tilde{b}_{j}^{0}\right| \geq \frac{1}{2}\right) \\
& =\operatorname{prob}\left(\left|\sum_{j \in \mathcal{N}_{i}}\left[\hat{b}_{j}^{0}(K)-\tilde{b}_{j}^{0}\right]\right| \geq \frac{1}{2}\right) \\
& \leq 4 \sum_{j \in \mathcal{N}_{i}} E\left[\left(\hat{b}_{j}^{0}(K)-\tilde{b}_{j}^{0}\right)^{2}\right]=4 \sum_{j \in \mathcal{N}_{i}} \zeta_{j, i}(K),
\end{aligned}
$$

where in the last line we are using Chebyshev's inequality. Furthermore, we have,

$$
\zeta_{j, i}(K)=\frac{1}{\frac{1}{\tilde{q}_{j}^{0}\left(1-\tilde{q}_{j}^{0}\right)}+\sum_{k=0}^{K} \mathrm{CNR}_{j, i}(k)}<\gamma_{j, i}^{-1}(K)
$$

where $\gamma_{j, i}(K) \triangleq \sum_{k=0}^{K} \operatorname{CNR}_{j, i}(k)$. Since $\operatorname{CNR}_{j, i}(k) \mathrm{s}$ are i.i.d. exponential random variables with the mean of $\overline{\mathrm{CNR}}$, $\gamma_{j, i}(K)$ has Gamma distribution with the following parameters: $\gamma_{j, i}(K) \sim \operatorname{Gamma}(K+1, \overline{\mathrm{CNR}})$. As a result, $\gamma_{j, i}^{-1}(K)$ is an inverse Gamma random variable. We then have, $\lim _{K \rightarrow \infty} \mathbf{E}\left\{\gamma_{j, i}^{-1}(K)\right\}=\lim _{K \rightarrow \infty} \frac{1}{(K+1) \times \overline{\mathrm{CNR}}}=0$. Since $\gamma_{j, i}^{-1}(K)$ is a non-negative random variable, we have

$$
\lim _{K \rightarrow \infty} \frac{1}{\gamma_{j, i}(K)}=0
$$

Therefore, $\lim _{K \rightarrow \infty} \zeta_{j, i}(K)=0$, which results in $\lim _{K \rightarrow \infty} \operatorname{prob}\left(b_{i}(K+1)=\tilde{b}_{i}^{1}\right)=1$ for $1 \leq i \leq M$.

Figure 2 shows the performance of both fusion and diversity strategies over a network of $M=17$ nodes that is trying to reach consensus by communicating over a regular ring lattice topology. Figure 2 (left) shows the comparison for a 16-regular ring topology, i.e., fully connected graph, where the transmissions occur over Rayleigh channels with $\overline{\mathrm{CNR}}=-6 \mathrm{~dB}$. Since the main bottleneck is the link qualities, the diversity approach outperforms the fusion approach and reaches accurate consensus asymptotically. The fusion strategy, on the other hand, has a 

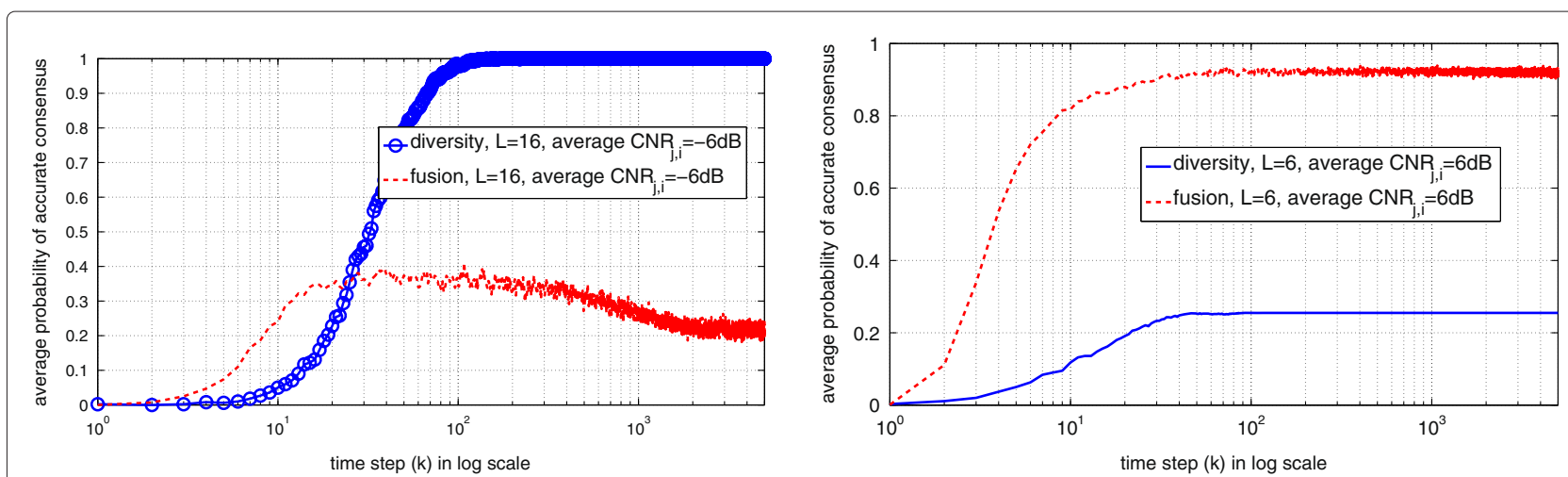

Figure 2 Performance comparison of fusion and diversity approaches for binary consensus over regular ring lattice topology. The $M=17$ nodes with fading channels and different graph connectivities: (left) $L=16$ and $\overline{\mathrm{CNR}}=-6 \mathrm{~dB}$, (right) $L=6$ and $\overline{\mathrm{CNR}}=6 \mathrm{~dB}$ (averaging is done over several runs)

better transient behavior. This is due to the fact that by fusing the received information, it helps in propagating the information over the network. However, it lacks asymptotic accurate consensus and suffers from asymptotic memoryless behavior. As the network connectivity decreases, the fusion strategy outperforms the diversity. For instance, Figure 2 (right) compares the performance of both approaches for 6-regular ring topology and $\overline{\mathrm{CNR}}=$ $6 \mathrm{~dB}$. Since the main bottleneck is the network connectivity, the fusion approach outperforms the diversity one drastically. In summary, the fusion strategy is more suitable when the graph connectivity is low, while the diversity technique is a better candidate if poor link quality is the main bottleneck. The analysis and simulation of this part show the underlying trade-offs between fusion and diversity strategies. In the next section, we propose a novel framework that keeps the benefits of both fusion and diversity approaches.

\section{An integrated diversity-fusion framework for binary consensus over fading channels}

In this section, we propose our diversity-fusion framework for binary consensus over fading channels. In this strategy, each agent sends a vector to its neighbors. This vector consists of the estimations of the votes corresponding to the different fusion levels. Throughout the repeated communications (diversity), each node tries to refine its assessments of different fusion levels in order to reach consensus.

Let $B_{i}(k)=\left[b_{i}^{0}(k), b_{i}^{1}(k), \ldots, b_{i}^{l_{G}(k)}(k)\right]^{T}$ represent the vector that node $i$ will send to all its neighbors over fading channels at time $k^{\mathrm{e}}$, where $b_{i}^{u}(k)$ represents the $i$ th node's estimate of $\tilde{b}_{i}^{u}$ at time $k$ and $l_{G}(k)=\min \left\{k, u_{G}-1\right\}$. Note that $\tilde{b}_{i}^{u}$ is defined in Section 2 as the value of node $i$ after $u$ fusion levels for the case where there is no noise. Table 1 shows the time progression of the transmitted vector by node $i$ to all its neighbors. As can be seen, at time $t=0$, node $i$ only transmits its initial vote to its neighbors. It also receives its neighbors' initial votes over fading channels. Next, in time step $t=1$, node $i$ fuses all its receptions in order to come up with an estimate of $\tilde{b}_{i}^{1}$. It then transmits its initial vote together with the estimate of $\tilde{b}_{i}^{1}$ to all its neighbors. This process will go on for a while, and each agent sends its estimate of different fusion levels to it neighbors. As can be seen, the $i$ th node's estimate of $\tilde{b}_{i}^{u}$ will not be available till $k=u$. Therefore, for $k<u_{G}-1, B_{i}(k)=\left[b_{i}^{0}(k), b_{i}^{1}(k), \ldots, b_{i}^{k}(k)\right]^{T}$ and $l_{G}(k)=k$. However, for $t \geq u_{G}-1$, the length of the transmitted vector becomes fixed and equal to $u_{G} .{ }^{f}$ It is worth noting that except for the first row, the columns of other rows have different values. This is due to the fact that similar transmissions can undergo different noise and fading values.

Let $r_{j, i}^{u}(k)$ and $n_{j, i}^{u}(k)$ represent the fading coefficient and the receiver noise of the link, which transmits $b_{j}^{u}(k)$ from node $j$ to node $i$, respectively. $n_{j, i}^{u}(k)$ is the zero-mean Gaussian with the variance of $\sigma_{j, i, u}^{2}$. We take the fading coefficient and the receiver noise of all the receptions to be uncorrelated. Let $b_{j, i}^{u}(k)$ represent the reception of the $i$ th node from the transmission of $b_{j}^{u}(k)$ at time $k$. We have the following, $b_{j, i}^{u}(k)=r_{j, i}^{u}(k) b_{j}^{u}(k)+n_{j, i}^{u}(k)$ for $1 \leq i \leq$ $M$ and $j \in \mathcal{N}_{i}$. Let $Z_{j, i}^{u}(k)=\left[b_{j, i}^{u}(u), \ldots, b_{j, i}^{u}(k)\right]^{T}$ represent all the receptions of node $i$ from node $j$ corresponding to

Table 1 Time progression of transmitted vector by node $i$

\begin{tabular}{cccccc}
\hline $\boldsymbol{t}=\mathbf{0}$ & $\boldsymbol{t}=\mathbf{1}$ & $\cdots$ & $\boldsymbol{t}=\boldsymbol{u}_{\boldsymbol{G}} \mathbf{- 1}$ & $\cdots$ & $\boldsymbol{t}=\boldsymbol{k}$ \\
\hline$b_{i}^{0}(0)$ & $b_{i}^{0}(1)$ & $\cdots$ & $b_{i}^{0}\left(u_{G}-1\right)$ & $\cdots$ & $b_{i}^{0}(k)$ \\
& $b_{i}^{1}(1)$ & $\ldots$ & $b_{i}^{1}\left(u_{G}-1\right)$ & $\cdots$ & $b_{i}^{1}(k)$ \\
& & $\ldots$ & $\vdots$ & $\ldots$ & $\vdots$ \\
& & $\cdots$ & $b_{i}^{u_{G}-1}\left(u_{G}-1\right)$ & $\cdots$ & $b_{i}^{u_{G}-1}(k)$ \\
\hline
\end{tabular}


the $u$ th level of fusion till time $k$. Node $i$ will then update its estimate of $b_{i}^{u+1}$ at time $k+1$ based on $b_{i}^{u}(k)$ and all the $u$ th level votes of its neighbors until time $k$.

$$
b_{i}^{u+1}(k+1)=\mathcal{F}\left(b_{i}^{u}(k), Z_{j, i}^{u}(k) \forall j \in \mathcal{N}_{i}\right),
$$

where $\mathcal{F}$ represents a decision-making function. The redundancy in vector $Z_{j, i}^{u}(k)$ tries to improve the link qualities. We next show how to design this function. We define the following variables: $\hat{B}_{j}^{u}(k)=\left[b_{j}^{u}(u), \ldots, b_{j}^{u}(k)\right]^{T}, R_{j, i}^{u}(k)=\left[r_{j, i}^{u}(u), \ldots, r_{j, i}^{u}(k)\right]^{T}$, $H_{j, i}^{u}(k)=\operatorname{diag}\left(R_{j, i}^{u}(k)\right), N_{j, i}^{u}(k)=\left[n_{j, i}^{u}(u), \ldots, n_{j, i}^{u}(k)\right]^{T}$, where $N_{j, i}^{u}(k) \sim \mathcal{N}\left(0, \sigma_{j, i, u}^{2} I_{(k-u+1) \times(k-u+1)}\right)$ and $\operatorname{diag}($.$) is a diagonal matrix with the elements of the$ argument on its main diagonal. Therefore, we have $Z_{j, i}^{u}(k)=H_{j, i}^{u}(k) \hat{B}_{j}^{u}(k)+N_{j, i}^{u}(k)$ for $k \geq u$. The $i$ th node will then try to estimate $\tilde{b}_{j}^{u}$, based on all receptions from $j$ th node, i.e., $Z_{j, i}^{u}(k)$. In order to characterize the best affine estimation of $\tilde{b}_{j}^{u}$, the second-order statistic of $\hat{B}_{j}^{u}(k)$ is required. However, finding a closed-form expression for the second-order statistics of this variable is challenging. The vector $\hat{B}_{j}^{u}(k)$ contains different temporal assessments of the $j$ th node about $\tilde{b}_{j}^{u}$. To simplify the mathematical derivation, we consider the case, where the $u$ th level of fusion is in its steady state, i.e., $\hat{B}_{j}^{u}(k)=\tilde{b}_{j}^{u} \overrightarrow{1}_{k-u+1} \quad \forall j$. Under this assumption, the BAE estimation can be derived similar to Equation 6 as follows: $\hat{b}_{j}^{u}(k)=\rho_{j, i}^{u T}(k) Z_{j, i}^{u}(k)+\eta_{j, i}^{u}(k)$, where $\rho_{j, i}^{u}(k)=$ $\frac{1}{\frac{\sigma_{j, i, u}^{2}}{\bar{\tau}_{j\left(1-\tilde{q}^{u}\right)}}+\left\|R_{j, i}^{u}(k)\right\|^{2}} R_{j, i}^{u}(k), \eta_{j, i}^{u}(k)=\tilde{q}_{j}^{u}\left(1-\rho_{j, i}^{u T}(k) R_{j, i}^{u}(K)\right)$ and $\tilde{q}_{j}^{u}=\mathbf{E}\left[\tilde{b}_{j}^{u}\right]$. Since $\hat{B}_{j}^{u}(k) \neq \tilde{b}_{j}^{u} \overrightarrow{1}_{k-u+1}$, the estimator is suboptimal. However, in Theorem 1 , we will show that $\lim _{k \rightarrow \infty} b_{j}^{u}(k)=\tilde{b}_{j}^{u}$ for $1 \leq j \leq M$. Therefore, the proposed suboptimal estimator is asymptotically optimal. The $i$ th node will then update its $(u+1)$ th level decision as

$$
\begin{aligned}
b_{i}^{u+1}(k+1)= & \Upsilon_{\text {fading, }\left|\mathcal{N}_{i}\right|+1} \\
& \times\left(\frac{1}{\left|\mathcal{N}_{i}\right|+1}\left[b_{i}^{u}(k)+\sum_{j \in \mathcal{N}_{i}} \hat{b}_{j}^{u}(k)\right], b_{i}^{u}(k)\right) \\
= & \Upsilon_{\text {fading, }\left|\mathcal{N}_{i}\right|+1}\left(\frac { 1 } { | \mathcal { N } _ { i } | + 1 } \left[b_{i}^{u}(k)\right.\right. \\
& \left.\left.+\sum_{j \in \mathcal{N}_{i}} \rho_{j, i}^{u T}(k) Z_{j, i}^{u}(k)+\eta_{j, i}^{u}(k)\right], b_{i}^{u}(k)\right),
\end{aligned}
$$

for $k \geq u, 0 \leq u \leq u_{G}-1$ and $b_{i}^{0}(k)=\tilde{b}_{i}^{0}$ $\forall i, k$. Next we show that the decision-making function of Equation 12 achieves accurate consensus asymptotically and overcomes the asymptotic memoryless behavior of the traditional fusion approaches.
Theorem 1. Consider binary consensus over a timeinvariant network topology with i.i.d. Rayleigh fading channels. Then, the decision-making function of Equation 12 asymptotically converges (in probability) to accurate consensus if $D^{0} \in \mathcal{D}_{G}$.

Proof. We prove the theorem by induction. Define $\omega_{i}^{u}(k) \triangleq \operatorname{prob}\left(b_{i}^{u}(k) \neq \tilde{b}_{i}^{u}\right)$. For $u=0$, we have $b_{i}^{0}(k)=$ $\tilde{b}_{i}^{0} \quad \forall k, i$. From Lemma 1, it can be easily confirmed that $\lim _{k \rightarrow \infty} \omega_{i}^{1}(k)=0 \quad \forall i$. Assume $\lim _{k \rightarrow \infty} \omega_{i}^{u}(k)=0$. We next prove that $\lim _{k \rightarrow \infty} \omega_{i}^{u+1}(k+1)=0$.

Similar to Lemma 1 , we can show that if $\mid b_{i}^{u}(k)+$ $\sum_{j \in \mathcal{N}_{i}} \rho_{j, i}^{u T}(k) Z_{j, i}^{u}(k)+\eta_{j, i}^{u}(k)-\sum_{j \in \mathcal{N}_{i} \bigcup\{i\}} \tilde{b}_{j}^{u} \mid \leq \frac{1}{2}$, then we have $b_{i}^{u+1}(k+1)=\tilde{b}_{i}^{u+1}$. Hence,

$$
\begin{aligned}
& \omega_{i}^{u+1}(k+1) \\
& =\operatorname{prob}\left(b_{i}^{u+1}(k+1) \neq \tilde{b}_{i}^{u+1}\right) \\
& \leq \operatorname{prob}\left(\mid b_{i}^{u}(k)+\sum_{j \in \mathcal{N}_{i}} \rho_{j, i}^{u T}(k) Z_{j, i}^{u}(k)+\eta_{j, i}^{u}(k)\right. \\
& \left.-\sum_{j \in \mathcal{N}_{i} \bigcup\{i\}} \tilde{b}_{j}^{u} \mid \geq \frac{1}{2}\right) \\
& =\operatorname{prob}\left(\mid b_{i}^{u}(k)-\tilde{b}_{i}^{u}+\sum_{j \in \mathcal{N}_{i}} \frac{1}{\frac{\sigma_{j, i, u}^{2}}{\tilde{q}_{j}^{u}\left(1-\tilde{q}_{j}^{u}\right)}+\left\|R_{j, i}^{u}(k)\right\|^{2}}\right. \\
& \times\left(\sum_{t=u}^{k} r_{j, i}^{u 2}(t)\left(b_{j}^{u}(t)-\tilde{b}_{j}^{u}\right)+\frac{\sigma_{j, i, u}^{2}}{1-\tilde{q}_{j}^{u}}\left(1-\frac{\tilde{b}_{j}^{u}}{\tilde{q}_{j}^{u}}\right)\right. \\
& \left.\left.+R_{j, i}^{u T}(k) N_{j, i}^{u}(k)\right) \mid \geq \frac{1}{2}\right) \\
& \leq \operatorname{prob}\left(\left|b_{i}^{u}(k)-\tilde{b}_{i}^{u}\right|+\sum_{j \in \mathcal{N}_{i}} \frac{1}{\left\|R_{j, i}^{u}(k)\right\|^{2}}\right. \\
& \times\left(\sum_{t=u}^{k} r_{j, i}^{u 2}(t)\left|b_{j}^{u}(t)-\tilde{b}_{j}^{u}\right|+\frac{\sigma_{j, i, u}^{2}}{1-\tilde{q}_{j}^{u}}\left|1-\frac{\tilde{b}_{j}^{u}}{\tilde{q}_{j}^{u}}\right|\right. \\
& \left.\left.+\left|R_{j, i}^{u T}(k) N_{j, i}^{u}(k)\right|\right) \geq \frac{1}{2}\right),
\end{aligned}
$$

where in the last inequality, we are using the property that for any random variables $\Xi_{i}$, we have $\operatorname{prob}\left(\left|\sum_{i} \Xi_{i}\right| \geq\right.$ $\epsilon) \leq \operatorname{prob}\left(\sum_{i}\left|\Xi_{i}\right| \geq \epsilon\right)$. Furthermore, we have $\mathbf{E}\left\{\left|b_{i}^{u}(k)-\tilde{b}_{i}^{u}\right|\right\}=\omega_{i}^{u}(k)$. Applying Markov's inequality results in 


$$
\begin{aligned}
& \omega_{i}^{u+1}(k+1) \\
& \leq 2\left[\omega_{i}^{u}(k)+\sum_{j \in \mathcal{N}_{i}} \frac{1}{\left\|R_{j, i}^{u}(k)\right\|^{2}} \times\left(\sum_{t=u}^{k} r_{j, i}^{u}{ }^{2}(t) \omega_{j}^{u}(k)+\frac{\sigma_{j, i, u}^{2}}{1-\tilde{q}_{j}^{u}}\left|1-\frac{\tilde{b}_{j}^{u}}{\tilde{q}_{j}^{u}}\right|+\mathbf{E}\left\{\left|R_{j, i}^{u} T^{\prime}(k) N_{j, i}^{u}(k)\right|\right\}\right)\right] \\
& =2\left[\omega_{i}^{u}(k)+\sum_{j \in \mathcal{N}_{i}} \frac{1}{\left\|R_{j, i}^{u}(k)\right\|^{2}}\left(\sum_{t=u}^{k} r_{j, i}^{u 2}(t) \omega_{j}^{u}(k)+\frac{\sigma_{j, i, u}^{2}}{1-\tilde{q}_{j}^{u}}\left|1-\frac{\tilde{b}_{j}^{u}}{\tilde{q}_{j}^{u}}\right|\right)+\sqrt{\frac{2}{\pi}} \sum_{j \in \mathcal{N}_{i}} \frac{\sigma_{j, i, u}}{\left\|R_{j, i}^{u}(k)\right\|}\right] \\
& =2\left[\omega_{i}^{u}(k)+\sum_{j \in \mathcal{N}_{i}} \frac{1}{\left\|R_{j, i}^{u}(k)\right\|^{2}} \sum_{t=u}^{k} r_{j, i}^{u 2}(t) \omega_{j}^{u}(k)+\sum_{j \in \mathcal{N}_{i}} \frac{\left|1-\frac{\tilde{b}_{j}^{u}}{\tilde{q}_{j}^{u}}\right|}{1-\tilde{q}_{j}^{u}} \frac{1}{\gamma_{j, i}^{u}(k)}+\sqrt{\frac{2}{\pi}} \sum_{j \in \mathcal{N}_{i}} \frac{1}{\sqrt{\gamma_{j, i}^{u}(k)}}\right],
\end{aligned}
$$

where $\gamma_{j, i}^{u}(k)=\sum_{t=u}^{k} \operatorname{CNR}_{j, i}^{u}(t)=\frac{\left\|R_{j, i}^{u}(k)\right\|^{2}}{\sigma_{j, i, u}^{2}}$. Furthermore, for the last term in the third line, we are using the property that if $\Xi$ is a zero-mean Gaussian variable with the variance of $\sigma^{2}$, then $\mathbf{E}\{\sqrt{\Xi}\}=\sqrt{\frac{2}{\pi}} \sigma$. Let $\epsilon>0$. Since $\lim _{k \rightarrow \infty} \omega_{j}^{u}(k)=0$, there exists a number $T_{j}^{u}(\epsilon)$ such that $\omega_{j}^{u}(k)<\epsilon$ for all $k \geq T_{j}^{u}(\epsilon)$. Therefore,

$$
\begin{aligned}
\frac{1}{\left\|R_{j, i}^{u}(k)\right\|^{2}} \sum_{t=u}^{k} r_{j, i}^{u 2}(t) \omega_{j}^{u}(t) \leq & \frac{1}{\left\|R_{j, i}^{u}(k)\right\|^{2}} \sum_{t=u}^{T_{j}^{u}(\epsilon)} r_{j, i}^{u 2}(t) \omega_{j}^{u}(t) \\
& +\frac{\left\|R_{j, i}^{u}(k)\right\|^{2}-\left\|R_{j, i}^{u}\left(T_{j}^{u}(\epsilon)\right)\right\|^{2}}{\left\|R_{j, i}^{u}(k)\right\|^{2}} \epsilon \\
\leq & \frac{1}{\left\|R_{j, i}^{u}(k)\right\|^{2}} \sum_{t=u}^{T_{j}^{u}(\epsilon)} r_{j, i}^{u 2}(t) \omega_{j}^{u}(t)+\epsilon .
\end{aligned}
$$

Since $r_{j, i}^{u}(t)^{\prime}$ s are i.i.d. random variables, we have $\mathbf{E}\left\{\frac{r_{j, i}^{u}(t)}{\left\|R_{j, i}^{u}(k)\right\|^{2}}\right\}=\frac{1}{k-u+1}$. Therefore,

$$
\lim _{k \rightarrow \infty} \mathbf{E}\left\{\frac{\sum_{t=u}^{T_{j}^{u}(\epsilon)} r_{j, i}^{u 2}(t)}{\left\|R_{j, i}^{u}(k)\right\|^{2}}\right\}=\lim _{k \rightarrow \infty} \frac{T_{j}^{u}(\epsilon)-u+1}{k-u+1}=0
$$

Since $\frac{1}{\left\|R_{j, i}^{u}(k)\right\|^{2}} \sum_{t=u}^{T_{j}^{u}(\epsilon)} r_{j, i}^{u 2}(t)$ is a non-negative random variable, it goes to 0 as $k \rightarrow \infty$. Therefore, we have, $\lim _{k \rightarrow \infty} \frac{1}{\left\|R_{j, i}^{u}(k)\right\|^{2}} \sum_{t=u}^{k} r_{j, i}^{u 2}(t) \omega_{j}^{u}(t)=0$. Furthermore, similar to Equation 10, we can show that $\lim _{k \rightarrow \infty} \frac{1}{\gamma_{j, i}^{u}(k)}=$ 0 . By substituting these values in Equation 13, it can be easily confirmed that $\lim _{k \rightarrow \infty} \omega_{i}^{u+1}(k+1)=0$. Using induction, we have $\lim _{k \rightarrow \infty} \omega_{i}^{u_{G}}(k)=0$ and as a result $\lim _{k \rightarrow \infty} \operatorname{prob}\left(b_{i}^{u_{G}}(k)=\tilde{b}_{i}^{u_{G}}\right)=1$ for $1 \leq i \leq M$. Therefore, if $D^{0} \in \mathcal{D}_{G}$, then $\lim _{k \rightarrow \infty}\left[b_{1}^{u_{G}}(k), \cdots, b_{M}^{u_{G}}(k)\right]$ is an accurate consensus state with the probability of one, which proves the theorem.
Remark 1. The decision-making function of Equation 12 uses information of $\sigma_{j, i, u}$ s. If such information is not available, then the $i$ th node can estimate $\tilde{b}_{j}^{u}$ using best linear unbiased estimation: $\hat{b}_{j}^{u}(k)=\frac{1}{\left\|R_{j, i}^{u}(k)\right\|^{2}} R_{j, i}^{u T}(k) Z_{j, i}^{u}(k)$, assuming that the $u$ th level of fusion is in its steady state. Each node will then update its $(u+1)$ th level decision as follows:

$$
\begin{aligned}
b_{i}^{u+1}(k+1)= & \Upsilon_{\text {fading, }\left|\mathcal{N}_{i}\right|+1} \\
& \times\left(\frac{1}{\left|\mathcal{N}_{i}\right|+1}\left[b_{i}^{u}(k)+\sum_{j \in \mathcal{N}_{i}} \hat{b}_{j}^{u}(k)\right], b_{i}^{u}(k)\right) \\
= & \Upsilon_{\text {fading, }\left|\mathcal{N}_{i}\right|+1}\left(\frac { 1 } { | \mathcal { N } _ { i } | + 1 } \left[b_{i}^{u}(k)\right.\right. \\
& \left.\left.+\sum_{j \in \mathcal{N}_{i}} \frac{1}{\left\|R_{j, i}^{u}(k)\right\|^{2}} R_{j, i}^{u T}(k) Z_{j, i}^{u}(k)\right], b_{i}^{u}(k)\right)
\end{aligned}
$$

for $k \geq u, 0 \leq u \leq u_{G}-1$, and $b_{i}^{0}(k)=\tilde{b}_{i}^{0} \forall i, k$. This receiver can be considered as a special case of the decision-making function of Equation 12, where $\sigma_{j, i, u}=0$. We can similarly show that it achieves accurate asymptotic consensus. Furthermore, we have $\lim _{k \rightarrow \infty} \rho_{j, i}^{u}(k) \approx$ $\frac{1}{\left\|R_{j, i}^{u}(k)\right\|^{2}} R_{j, i}^{u}(k)$ and $\lim _{k \rightarrow \infty} \eta_{j, i}^{u}(k) \approx 0$.

In the subsequent sections, we utilize the integrated framework for a special class of undirected graphs and show the performance of the proposed framework.

\section{Integrated framework over regular ring lattice topologies}

In Section 4, we introduced our proposed framework, which asymptotically achieves accurate consensus. In this approach, node $i$ will send a vector $B_{i}(k)$ to all its neighbors over fading channels. The length of this vector is $u_{G}$ for $t \geq u_{G}-1$, which is a function of graph connectivity. Intuitively, networks with higher connectivity require smaller values of $u_{G}$. For instance, for fully connected 
networks, we have $u_{G}=1$. In general, $u_{G}$ is a function of network topology and independent of communication quality. This parameter needs to be determined before running the algorithm. In this section, we mainly focus on $L$-regular ring lattice topologies with ideal communication links in order to characterize $u_{G}$. Let $\mathcal{V}=$ $\{1,2, \ldots, M\}$ denote the vertex set. Without loss of generality, we assume that the vertices are ordered clockwise on the ring (see Figure 1). Furthermore, we assume that $M$ is odd. Under the above assumptions, the adjacency matrix of an $L$-regular ring lattice, i.e., $A_{L}$, can be represented by a circulant matrix with the first row of $\left[0, \overrightarrow{1}_{\frac{L}{2}}^{T}, \overrightarrow{0}_{M-L-1}^{T}, \overrightarrow{1}_{\frac{L}{2}}^{T}\right]$. For this class of graphs, $L$ is a notion of connectivity. Therefore, we try to show how $u_{G}$ changes as a function of $L$. We then have the following lemma, which will be used in Theorem 2.

Lemma 2. For an L-regular ring lattice, if $L=M-1$, then $\left|\cap_{i=1}^{M}\left\{\mathcal{N}_{i} \cup\{i\}\right\}\right|=M$. Moreover, if $L \leq M-3$, then $\left|\cap_{n=1}^{p}\left\{\mathcal{N}_{i_{n}} \cup\left\{i_{n}\right\}\right\}\right| \leq L+2-p$. The equality is achieved if and only if $\left\{i_{1}, \ldots, i_{p}\right\} \subset \mathcal{V}$ denotes a set of consecutive nodes on the corresponding ring.

Proof. The proof is straightforward and we skip it.

Let $m^{u}=\min \left(\sum_{j=1}^{M} \tilde{b}_{j}^{u}, M-\sum_{j=1}^{M} \tilde{b}_{j}^{u}\right)$. We then have the following theorem.

Theorem 2. Consider binary consensus over an L-regular ring lattice. We then have the following properties:

1. Assume $\mathcal{V}=\bigcup_{i=1}^{c} \mathcal{V}_{i}$, such that $\mathcal{V}_{i}$ represents a set of consecutive nodes on the ring that vote the same and $\left|\mathcal{V}_{i}\right| \geq \frac{L}{2}+1$. Then the corresponding network state is an absorbing state.

2. For $m^{0} \leq \frac{L}{2}$, the accurate consensus is achievable after one level of fusion.

3. For $m^{0}=\frac{L}{2}+1$ and $D^{0} \in \mathcal{D}_{G}$, accurate consensus is achievable at most after two fusion steps.

Proof. Let $\mathcal{V}_{i}=\left\{o_{1}^{i}, \ldots, o_{\left|\mathcal{V}_{i}\right|}^{i}\right\}$ denote the $i$ th partition, where $o_{j}^{i}$ represents the index of $j$ th node in the $i$ th partition. Therefore, we have, $\left|\left\{\mathcal{N}_{o_{j}^{i}} \cup\left\{o_{j}^{i}\right\}\right\} \cap \mathcal{V}_{i}\right| \geq \frac{L}{2}+1$ for all $1 \leq j \leq\left|\mathcal{V}_{i}\right|$. Since all the nodes in $\mathcal{V}_{i}$ vote the same, we have $\overline{\tilde{b}}_{o_{j}^{i}}^{u+1}=\tilde{b}_{o_{j}^{i}}^{u}$ for $1 \leq i \leq c$ and $1 \leq j \leq\left|\mathcal{V}_{i}\right|$.

We next prove the second part. Consider the case where the majority of the initial votes is 1 . If $m^{0} \leq \frac{L}{2}$, we then have $\frac{\tilde{b}_{i}^{0}+\sum_{j \in \mathcal{N}_{i}} \tilde{b}_{j}^{0}}{L+1} \geq \frac{L+1-m^{0}}{L+1}>\frac{1}{2} \forall i$, which results in $\tilde{b}_{i}^{1}=1$. For the case where the majority of the initial votes is 0 , we have $\frac{\tilde{b}_{i}^{0}+\sum_{j \in \mathcal{N}_{i}} \tilde{b}_{j}^{0}}{L+1} \leq \frac{m^{0}}{L+1}<\frac{1}{2} \forall i$, which results in $\tilde{b}_{i}^{1}=0$. Therefore, for $m^{0} \leq \frac{L}{2}$ accurate consensus is achievable in one iteration.

Next, we show the third statement. First we show that for $m^{0}=\frac{L}{2}+1$ and $D^{0} \in \mathcal{D}_{G}$, we have $m^{1} \leq \frac{L}{2}$. Let $\left\{i_{1}^{0}, i_{2}^{0}, \ldots, i_{m^{0}}^{0}\right\}$ denote an ordered set of the nodes, which vote to the minority of the initial votes. Lemma 2 says that at most $L+2-m^{0}=\frac{L}{2}+1$ nodes can have $\left\{i_{1}^{0}, \ldots, i_{m^{0}}^{0}\right\}$ in their neighbor set if and only if $\left\{i_{1}^{0}, i_{2}^{0}, \ldots, i_{m^{0}}^{0}\right\}$ is a set of consecutive vertices. Therefore, $m^{1}=\frac{L}{2}+1$ is achievable if and only if the initial state is an absorbing state (see Theorem 2-1). Therefore, if $D^{0} \in \mathcal{D}_{G}$, then $m^{1} \leq \frac{L}{2}$, which reaches accurate consensus in $u=2$ (see Theorem 2-2).

In Theorem 2, we showed that the number of fusion levels, required to achieve accurate consensus is a function of graph connectivity $(L)$ and initial state $\left(D^{0}\right)$. For instance, for a fixed connectivity, if $m^{0} \leq \frac{L}{2}$, accurate consensus is achievable after one level of fusion. However, if $m^{0}$ increases to $\frac{L}{2}+1$, then the network may require two fusion steps to reach consensus. In order to understand the impact of the connectivity on the required fusion steps, Figure 3 characterizes $u_{G}$ as a function of $L$ over a regular ring lattice topology for different number of nodes. As can be seen, as $L$ increases, $u_{G}$ decreases. For instance, for $L=16$, i.e., a fully connected graph, the network requires only one level of fusion to reach accurate consensus. It can also be seen that $u_{G}$ is only a function of the graph topology. Figure 3 also compares $u_{G}$ for different number of nodes. As can be seen, $u_{G}$ is a non-decreasing function of $M$ for a fixed $L$. However, the mathematical derivations are challenging. Intuitively, as $M$ increases, the network requires more number of fusion levels, i.e., 'a higher' $u_{G}$, in order to achieve consensus. For instance, consider an $L$-regular ring lattice network of $M_{1}$ nodes, where $L=M_{1}-1$ and $M_{1}$ is an odd number. Since we always have $m^{0} \leq \frac{L}{2}=\frac{M_{1}-1}{2}$, therefore the network achieves accurate consensus only in one iteration. Next,

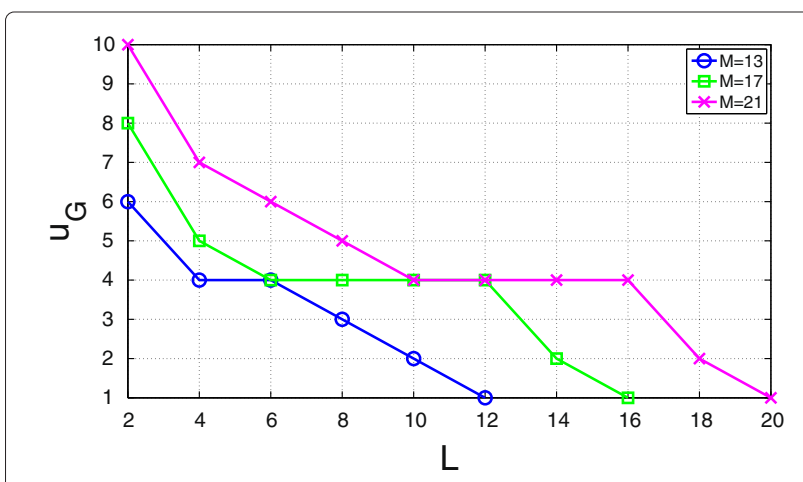

Figure $3 u_{G}$ as a function of $L$ for the regular ring lattice of $M=13,17$, and 21 nodes. 
consider an $L$-regular ring lattice network of $M_{2}$ nodes, where $L=M_{1}-1, M_{2}>M_{1}$ and $M_{2}$ is an odd number. It is easy to confirm that $u_{G} \geq 2$ in this case.

\section{Simulation and comparison}

Figure 4 shows the performance of the proposed framework over a regular ring lattice topology of $M=17$ nodes. In order to show the impact of network connectivity on the performance of the proposed algorithm, we consider a regular graph topology with different values of $L$. For each $L$, the number of required fusion levels, i.e., $u_{G}$, can be found from Figure 3 . Each node will then send a binary vector of length $u_{G}$ to all its neighbors over communication channels. Moreover, we assume all channels experience the same noise variance $\left(\sigma_{j, i, u}=1.5\right)$ and the average power of fading coefficients is equal to one $\left(E\left[r_{j, i}^{u 2}(k)\right]=1\right)$. In order to make a fair comparison (the proposed integrated approaches send more bits per iteration), we compare all the approaches under the same number of transmitted bits. Therefore, in Figure 4, we show the probability of accurate consensus as a function of number of transmitted bits per node.

Figure 4 shows the performance of the proposed framework for $L=8,10$, and 14. For these simulations, we assume that node $i$ does not have the knowledge of $q_{j}^{u}$ for $j \in \mathcal{N}_{i}$. So it simply assumes $q_{j}^{u}=0.5$ in the decision-making function of Equation 12. As can be seen, the the integrated approach, independent of network connectivity, achieves accurate consensus asymptotically. Therefore, the proposed approach overcomes the memoryless asymptotic behavior of traditional binary consensus approaches. Furthermore, networks with higher connectivity, i.e., larger values of $L$, reach their steady state in fewer transmissions. For the case of $L=8$, the performance of both fusion and diversity approaches of Section 3 are also shown for comparison. As can be seen,

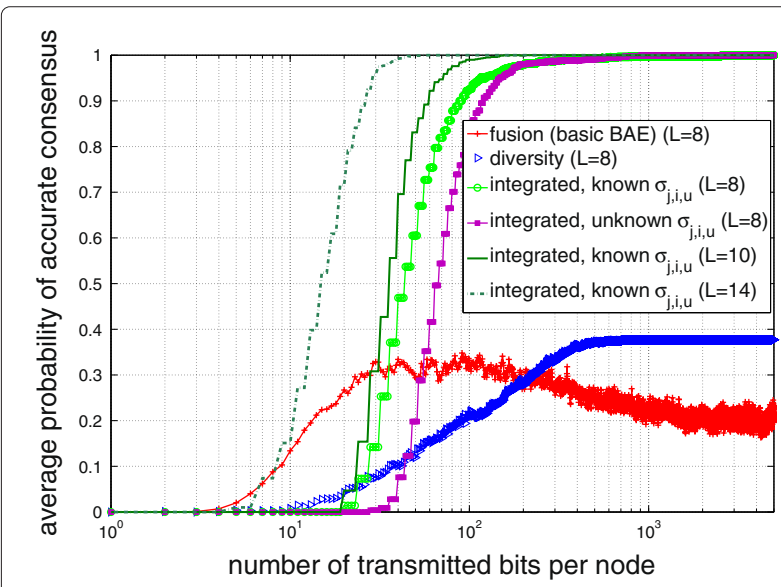

Figure 4 Average probability of accurate consensus over regular ring lattice of 17 nodes where $\overline{\mathrm{CNR}}=-3.5 \mathrm{~dB}$. the proposed approach keeps the benefits of both fusion and diversity in terms of the transient and asymptotic behaviors respectively. Furthermore, the performance of the integrated approach for the case where $L=8$ and knowledge of $\sigma_{j, i, u}$ is not available (Remark 1) is also shown in Figure 4. As can be seen, the integrated approach with known link quality slightly outperforms the case of unknown link quality. However, both cases provide a similar performance asymptotically as mentioned earlier in Remark 1.

\section{Conclusions}

In this paper we considered a cooperative network that is trying to reach binary consensus over not fully connected and time-invariant network topologies with fading channels. We first characterized the underlying trade-offs between fusion and diversity strategies. We then proposed a framework that keeps the benefits of both strategies. We mathematically analyzed the proposed algorithm and showed how it achieves accurate consensus asymptotically. Our results indicated that the proposed technique improves the consensus performance considerably and solves the undesirable memoryless asymptotic behavior of the original problem.

\section{Endnotes}

a We also use the term 'agent' to refer to each node.

b Note that in a binary consensus scenario, each node only exchanges 1 bit of information with its neighbors in each time step.

c An $L$-regular ring lattice is an $L$-regular graph with $M$ vertices in a ring in which each vertex is connected to its $L$ neighbors ( $\frac{L}{2}$ on each side for an even value of $L$ ).

d Note that without loss of generality, we assumed that the modulation is on-off keying.

e We assume an FDMA- or TDMA-based MAC approach. For instance, in FDMA-base approach, to each node we assign different frequency subbands, corresponding to different fusion levels.

${ }^{\mathrm{f}}$ Note that we chose $u_{G}$ as the maximum length based on the fact that, in the case of no noise, this is the number of fusion levels needed for convergence. However, in this case that we have noise, this may not be the optimum length and choosing longer lengths can possibly result in a better performance.

Competing interests

The authors declare that they have no competing interests.

Received: 22 April 2012 Accepted: 19 June 2013

Published: 4 September 2013

\section{References}

1. A Jadbabaie, J Lin, AS Morse, Coordination of groups of mobile autonomous agents using nearest neighbor rules. IEEE Trans. Automatic Control. 48, 988-1001 (2003) 
2. TC Aysal, ME Yildiz, AD Sarwate, A Scaglione, Broadcast gossip algorithms for consensus. IEEE Trans. Signal Process. 57, 2748-2761 (2009)

3. S Kar, J Moura, Distributed consensus algorithms in sensor networks with imperfect communication: Link failures and channel noise. IEEE Trans. Signal Process. 57, 355-369 (2009)

4. G Scutari, S Barbarossa, Distributed consensus over wireless sensor networks affected by multipath fading. IEEE Trans. Signal Process. 56, 4100-4106 (2008)

5. Y Mostofi, Binary consensus with Gaussian communication noise: a probabilistic approach, in Proceedings of the 46th IEEE Conference on Decision and Control (CDC). Hilton New Orleans Riverside, New Orleans, 12-14, Dec 2007

6. S Ashrafi, M Malmirchegini, Y Mostofi, Binary consensus for cooperative spectrum sensing in cognitive radio networks. IEEE GLOBECOM, Houston, 5-9 Dec 2011 (IEEE, Piscataway, 2011)

7. Y Hong, A Scaglione, P Varshney, A communication architecture for reaching the consensus in decision for a large network. IEEE Statistical Signal Processing Workshop, SSP2005, Bordeaux, 17-20 July 2005 (IEEE, Piscataway, 2005), pp. 17-22

8. R Olfati-Saber, E Franco, E Frazzoli, JS Shamma, Belief consensus and distributed hypothesis testing in sensor networks. Network Embedded Sensing Control. 331, 169-182 (2006)

9. J Liu, V Yadav, H Sehgal, J Olson, H Liu, N Elia, Phase transitions on fixed connected graphs and random graphs in the presence of noise. Proceedings of the 44th IEEE Conference on Decision and Control, Orlando, 12-15 Dec 2005 (IEEE, Piscataway, 2005), pp. 2996-3000

10. M Malmirchegini, Y Ruan, Y Mostofi, Binary consensus over fading channels: a best affine estimation approach. IEEE Global Telecommunications Conference, New Orleans, 30 Nov-4 Dec 2008 (IEEE, Piscataway, 2008)

11. Y Mostofi, M Malmirchegini, Binary consensus over fading channels. IEEE Trans. Signal Process. 58, 6340-6354 (2010)

doi:10.1186/1687-6180-2013-144

Cite this article as: Malmirchegini and Mostofi: An integrated diversity and fusion framework for binary consensus over fading channels. EURASIP Journal on Advances in Signal Processing 2013 2013:144.

\section{Submit your manuscript to a SpringerOpen ${ }^{\circ}$ journal and benefit from:}

- Convenient online submission

- Rigorous peer review

- Immediate publication on acceptance

- Open access: articles freely available online

- High visibility within the field

- Retaining the copyright to your article

Submit your next manuscript at $\boldsymbol{\triangleright}$ springeropen.com 